\title{
Integrating Cdk signaling in Candida albicans environmental sensing networks
}

\author{
Carlos R. Vázquez de Aldana ${ }^{1}$ and Jaime Correa-Bordes ${ }^{2}$ \\ ${ }^{1}$ Instituto de Biología Funcional y Genómica. CSIC-Universidad de Salamanca. Salamanca. \\ Spain; ${ }^{2}$ Departamento Ciencias Biomédicas. Universidad de Extremadura. Badajoz. Spain.
}

\begin{abstract}
Cyclin-dependent protein kinases (Cdks) control cell cycle progression and morphological switches in eukaryotic cells. Based on recent findings concerning the evolution of Cdk phosphorylation sites in the Ascomycete linage, we shall analyze the density of Cdk motifs in the Candida proteome using the $\mathrm{S}_{\mathrm{LR}}$ algorithm, focusing on protein sequences of regulatory modules that play important roles in the environmental sensing of C. albicans. Since Cdks are also involved in morphogenesis and environmental signaling, this search could help us to speculate about how Cdk signaling might be integrated in these regulatory networks that control C. albicans morphopathogenic determinants.
\end{abstract}

\section{Table of contents}

Integrating Cdk signaling in Candida albicans environmental sensing networks 1

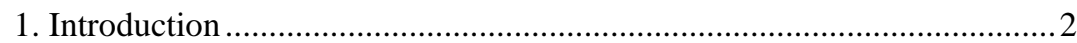

2. Evolution of phosphoregulation ................................................................ 3

3. Cyclin-dependent kinases in C. albicans .................................................. 4

4. Predicting Cdk targets in C. albicans proteins........................................ 5

4.1. Lessons from $S$. cerevisiae .......................................................... 5

4.2. Prediction of Cdk targets in the C. albicans proteome ..................... 6

4.2.1. Transcriptional regulation ......................................................... 8

4.2.2. Cell wall regulation.................................................................... 8

4.2.3. Cell signaling ......................................................................... 9

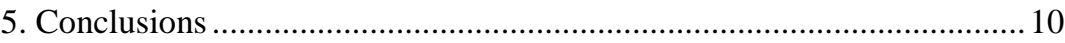

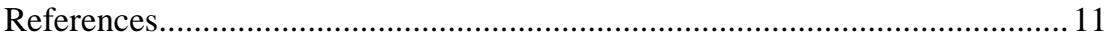




\section{Introduction}

In eukaryotic cells, global regulatory networks control cell physiology in response to external and internal cues. Many of these signaling networks are highly modular, making them more evolvable and providing increased fitness in competitive and changing environments (Bhattacharyya et al. 2006). Fungi are able to colonize and occupy highly divergent niches, ranging from high osmotic environments to plants and mammalian hosts. Their ability to adapt to these hostile environments depends on their capability to sense a variety of external cues, transduce the signals to specific cytoplasmic targets, and activate the appropriate responses.

Unlike the majority of fungi, the Ascomycete Candida albicans sis normally found as a commensal in the gastrointestinal tract of humans and warm-blooded animals. Although generally asymptomatic, C. albicans can cause mucosal infections in healthy people. In patients with a deficient immune system, such as HIVcompromised individuals or patients treated with immunosuppressive drugs after organ transplantation, this yeast can produce systemic infections in which the fungus can spread to all major organs of the body, leading to death in around 50\% of bloodstream infections (Eggimann et al., 2003; Gudlaugsson et al., 2003).

C. albicans has several attributes that allow it to adapt rapidly to changing environmental signals that contribute to host colonization. One of those best studied is the ability to switch between different morphologies, such as yeast, pseudohyphae and hyphae (Sudbery et al. 2004). The yeast-to-hypha transition, triggered by a wide range of environmental cues, is regulated by multiple signaling pathways that control the transcription of a set of hypha-specific genes (HSGs), many of which encode known virulence factors (Biswas et al. 2007; Calderone and Fonzi 2001; Whiteway and Bachewich 2007). The promoter regions of HSGs integrate the signals of multiple activators and repressors, although the interplay between them remains largely unknown. The two major transcriptional activators are Cph1 and Efg1 (Liu et al. 1994; Stoldt et al. 1997), which act downstream of the MAPK

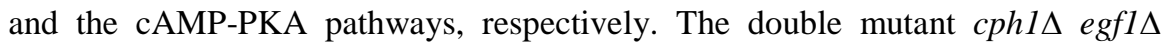
blocks hyphal transitions under most conditions tested and shows reduced virulence (Lo et al. 1997). Negative regulation of hyphal growth is achieved through the combinatorial association of the Tup1 repressor with the DNA-binding proteins (DBPs) Nrg1, Rfg1 and Mig1 (Braun et al. 2000; Braun and Johnson 1997; Kadosh and Johnson 2005; Murad et al. 2001a; Murad et al. 2001b). Cells depleted of any of these repressors are able to activate hyphal growth under yeastgrowth conditions. Since Mig1-Tup1 regulate a set of genes other than those regulated by Nrg1-Tup1, it has been suggested that DBPs target Tup1 to specific subsets of genes. However, Mig1 and Nrg1 can also repress the expression of other genes in a Tup1-independent manner (Murad et al. 2001a), suggesting that the DBPs associated with Tup1, (rather than Tup1 itself) are likely to be regulated 
during the yeast-to-hypha transition (Braun et al. 2000; Braun and Johnson 1997; Kadosh and Johnson 2005; Murad et al. 2001a; Murad et al. 2001b).

Another example of the enormous plasticity of $C$. albicans cells is whiteopaque (W/O) switching. This fungus undergoes an epigenetic switch between two cell types, known as white and opaque (Slutsky et al. 1987). These cell types differ in their cell morphology, metabolic state and mating behavior, and in their ability to form biofilms, their preferred niches in the host and interactions with the immune system. In recent years, new insights into the mechanisms that control this transition have allowed researchers to uncover complex relationships that relate switching, mating and pathogenesis (Lohse and Johnson 2009; Soll 2009).

Although Saccharomyces cerevisiae and C. albicans share some common features, they also exhibit many significant differences since they diverged from a common ancestor more than 500 million years ago. The shared evolutionary fate of $C$. albicans and its hosts might have allowed it to evolve several developmental programs that would be activated by environmental cues within the host and that could aid in the colonization of different niches. These colonization sites represent different environments in terms of cohabitant microbiota, $\mathrm{pH}$, nutrients and $\mathrm{O}_{2}$ or $\mathrm{CO}_{2}$ levels. Thus, this distinct life style might have been a driving force in rewiring C. albicans signaling networks ( $\mathrm{Li}$ and Johnson 2010).

\section{Evolution of phosphoregulation}

Protein phosphorylation is a ubiquitous and reversible modification that is crucial for the regulation of cellular events (Seet et al. 2006). Comparative studies of the phosphoproteome of three yeast species (C. albicans, S. cerevisiae and Schizosaccharomyces pombe) suggest that protein kinases probably contribute to a substantial extent to the evolution and generation of phenotypic diversity (Beltrao et al. 2009).

An important feature of C. albicans is that the CUG codon is decoded as Ser instead of Leu (Santos and Tuite 1995), with an average frequency of 1-6 CUGs per gene (Butler et al. 2009; Massey et al. 2003). Since Ser is a substrate of protein kinases, this change in codon usage might increase the number of potential phosphorylation sites per protein, adding new possible layers of phosphoregulation to protein networks. In fact, the ratio of phosphoserines/total proteins determined by mass spectrometry (MS) analysis in C. albicans is higher (0.54) than in S. cerevisiae (0.39) and S. pombe (0.32) (Beltrao et al. 2009).

Protein kinases regulate the function of their target proteins by adding a phosphate group to specific sites, which can change the activity of the protein through two different mechanisms (Holt et al. 2009). First, phosphorylation could drive a precise conformational change in the structure of the protein because the phosphate modifies the network of hydrogen bonds of several neighboring amino acids. This type of regulation, common in metabolic enzymes, is highly context- 
dependent and exhibits strong evolutionary conservation. Alternatively, the addition of phosphates to disordered regions (either the N- or C-termini or internal loops) of substrates can modify their interaction with other proteins (Serber and Ferrell 2007; Strickfaden et al. 2007) or can create new interactions through the phosphopeptide-binding modules present in other molecules, such as the SH2, 143-3, or WW domains (Bhattacharyya et al. 2006; Morrison 2009; Seet et al. 2006). In these cases, the position of the phosphoacceptor residue(s) is less contextdependent and therefore it can undergo a higher rate of change and a greater potential to generate functional diversity (Beltrao and Serrano 2007).

Cyclin-dependent protein kinases (Cdks) control progression along the eukaryotic cell cycle. These proteins are proline-directed kinases that preferentially phosphorylate substrates with the full consensus sequence S/T-P-X-K/R (where X is any amino acid), although they can also phosphorylate the minimal consensus sequence S/T-P (Echalier et al. 2010; Songyang et al. 1994). In this chapter, based on recent findings concerning the evolution of Cdk phosphorylation sites in the Ascomycete linage (Holt et al. 2009), we analyze the density of Cdk motifs in the C. albicans proteome using the $\mathrm{S}_{\mathrm{LR}}$ algorithm (Moses et al. 2007a) and focusing on proteins that are components of regulatory modules that play important roles in environmental sensing. Since Cdks are also involved in morphogenesis and environmental signaling (Huang et al. 2007; Moseley and Nurse 2009; Wang 2009), this search could help us to speculate about how Cdk signaling might be integrated in the regulatory networks that control morphopathogenic determinants in C. albicans.

\section{Cyclin-dependent kinases in C. albicans}

In yeast, cell cycle progression is driven by a single Cdk1 (Cdc28 in S. cerevisiae and C. albicans, Cdc2 in S. pombe). The combinatorial association of Cdk1 with G1 or G2 cyclins is thought to generate Cdk complexes with different substrate specificities that regulate different cell cycle transitions (Bloom and Cross 2007; Loog and Morgan 2005). However, phylogenetic studies of yeast B-type cyclins and experimental yeast models are consistent with the idea of an ancestral eukaryote with a single Cdk/cyclin module driving the cell cycle (Archambault et al. 2005; Coudreuse and Nurse 2010; Fisher and Nurse 1996). It is likely that the appearance of multiple cyclins in most eukaryotic lineages would have introduced new regulatory layers to fine-tune the single core Cdk module, providing more flexibility in the control of the cell cycle in response to different inputs (Bloom and Cross 2007; Coudreuse and Nurse 2010; Loog and Morgan 2005).

In addition to driving the cell cycle, Cdks coordinate cell morphology switches (Moseley and Nurse 2009; Wang 2009). In C. albicans, modifications of cyclin levels produce dramatic morphological changes. This fungus contains three G1 (Ccn1, Hgc1 and Cln3) and two G2 (Clb2 and Clb4) cyclins. Depletion of Cln3, 
Clb2 or Clb4 in yeast cells results in hyperpolarized growth in the absence of hypha-inducing conditions (Bachewich and Whiteway 2005; Bensen et al. 2005; Chapa y Lazo et al. 2005). Ccn1 is a non-essential G1 cyclin that is expressed during the G1/S transition and is required for the maintenance, but not the initiation, of hyphal growth under certain conditions (Loeb et al. 1999; Sinha et al. 2007). Hgc1 is a hypha-specific G1 cyclin-like protein that preferentially localizes to the dividing apical cell of the hyphae (Wang et al. 2007; Zheng and Wang 2004). Transcription from the HGC1 promoter is essential for this asymmetric cell localization, since Hgc1 no longer exhibits the preferential apical accumulation when expressed under the control of the MAL2 promoter (Wang et al. 2007). Deletion of HGC1 prevents hyphal growth under all hypha-inducing conditions and results in reduced virulence in mouse models (Zheng and Wang 2004). Unlike the cell-cycle regulated transcription of other cyclin genes, $\mathrm{HGC1}$ expression is activated by hypha-inducing signals through the CAMP/PKA signaling pathway. The evolution of such control would probably have been crucial for ensuring the cell-cycle independent polarized growth of hyphae (Wang 2009; Zheng and Wang 2004). In agreement with this hypothesis, the expression of one allele of CCN1 under the control of the HGC1 promoter rescues the $h g c 1 \Delta$ mutant (P. Gutiérrez-Escribano and J. Correa-Bordes, unpublished results).

In recent years, insight into the links between Cdks and cell polarity proteins has been obtained in C. albicans, highlighting the importance of Cdk1 complexes in the control of cell morphogenesis during yeast and hyphal growth (GonzálezNovo et al. 2008; Sinha et al. 2007; Wang et al. 2009; Zheng and Wang 2004; Zheng et al. 2007). An excellent summary of the role of Cdks in the yeast-hyphal transition of C. albicans has been published recently (Wang 2009).

\section{Predicting Cdk targets in C. albicans proteins}

Past efforts aimed at the identification of HSGs have underlined the importance of the cAMP-PKA and MAPK pathways in the transcriptional activation required for hyphal growth. However, recent findings have suggested that post-translational modifications mediated by Cdks are also important mechanisms in the regulation of polarized growth immediately after hyphal induction, independently of the cAMP-PKA and MAPK pathways (Sinha et al. 2007). These results suggest the existence of an additional signaling pathway(s) that plays a major role in the control of hyphal development, which is mediated by the Cdk-phosphorylation of key regulatory proteins in response to hypha-inducing signals.

\subsection{Lessons from $S$. cerevisiae}


Global analysis of the Cdk1-dependent $S$. cerevisiae phosphoproteome identified the position of 547 phosphorylation sites on 308 proteins, based on the specific chemical inhibition of Cdk1 and quantitative MS (Holt et al. 2009). Study of the structural context of the Cdk1 sites revealed some interesting features. First, more than $90 \%$ of the sites were located in loops and disordered regions. Second, Cdk1 substrates tended to be phosphorylated at multiple sites. Finally, Cdk1 phosphorylation sites tended to cluster in the primary sequence, suggesting that multiple phosphorylation events would modulate the same protein surface. Notably, comparisons of the substrates with their orthologs in another 32 Ascomycetes showed that the position of most of the phosphorylation sites was not highly conserved; instead, they shifted position inside the rapidly evolving disordered regions (Holt et al. 2009). In sum, although the minimal Cdk1 phosphorylation motif (S/T-P) is conserved over long evolutionary timescales, the regions containing them show rapid evolution. Thus, these features allow Cdk1 control mechanisms to adapt rapidly to developmental challenges that have arisen or may arise during the course of evolution. This flexibility in phosphorylation site positioning might have important biological implications, since the appearance of a linage-specific Cdk cluster in a protein could give rise to new regulatory controls. For example, it has been suggested that the Cdk-driven regulation of nuclear localization of the preReplicative Complex component Mcm3 could have appeared in the S. cerevisiae linage after its divergence from $C$. albicans through the acquisition of Cdk sites clustered at the ScMcm3 C-terminus (Moses et al. 2007b).

A new computational strategy aimed at identifying proteins that contain high densities of strong (S/T-P-X-R/K) and weak (S/T-P) Cdk consensus sites closely spaced in the amino acid sequence has recently been developed (Moses et al. 2007a). This method allows the identification of proteins in which Cdk clusters deviate from random expectation by calculating the likelihood ratio statistic $\left(\mathrm{S}_{\mathrm{LR}}\right)$. This cluster-based method measures the enrichment of motifs in a sequence and their spatial clustering. In order to define a $S_{\mathrm{LR}}$ cut-off value to use in the prediction of Cdk substrates, a comparison of the distribution of $\mathrm{S}_{\mathrm{LR}}$ scores using either the real Cdk consensus motifs or scrambled versions (P-R/K-X-S/T and P-S/T) was performed and a score threshold of 3.5 was defined. Therefore, cluster-based methods used in combination with other evidence, such as structural properties (Iakoucheva et al. 2004) or evolutionary conservation (Budovskaya et al. 2005), could be exploited to predict Cdk targets (Moses et al. 2007a).

\subsection{Prediction of Cdk targets in the C. albicans proteome}

Given the rapid evolution of Cdk phosphorylation site positioning in the disordered regions of proteins, we propose a speculative model whereby the commensal life style of $C$. albicans might have led Cdk evolution to be connected to a much broader range of signaling pathways than in $S$. cerevisiae. This would have allowed the integration of Cdk-control mechanisms with the different develop- 
mental programs triggered by environmental cues found in the host. This hypothesis would imply the existence of Candida linage-specific Cdk clusters in proteins involved in the response to environment signals, such as cell signaling proteins and transcriptional regulators. To test this hypothesis, we searched the C. albicans proteome for proteins containing putative regulatory Cdk clusters using the $S_{L R}$ algorithm. In order to reduce the number of false positives, a second criterion was used; this was that Cdk clusters had to be located in disordered regions, as determined by the PONDR algorithm (www.pondr.com). Finally, to test whether the identified putative regulatory clusters were linage-specific, we compared such regions with their ortholog proteins of other Hemiascomycota species

To identify putative Cdk targets in C. albicans, we analyzed the proteome using the $S_{L R}$ algorithm for the presence of clusters of strong and weak Cdk motifs and used a threshold $\mathrm{S}_{\mathrm{LR}}$ score of 3.5 (named $\mathrm{S}_{\mathrm{LRF}}$ analysis). This analysis identified 91 proteins with an $S_{L R}$ value above the cut-off, which represents the $1.46 \%$ of the total proteins (Fig. 1, inset). Of these 91 predictions, 52 of them had orthologs in S. cerevisiae. Gene Ontology (GO) analysis of the putative substrates revealed a strong enrichment for cell cycle-related functional categories (20/52; $38 \%)$. In addition, 34 of them (63\%) showed Cdk1-dependent phosphorylation in S. cerevisiae or C. albicans (Beltrao et al. 2009; Holt et al. 2009; Ubersax et al. 2003). Accordingly, the value of 3.5 seemed to be a good threshold when strong and weak Cdk motifs were used. However, given the existence of Cdk substrates lacking strong consensus sites regulated by Cdk phosphorylation at weak sites (Nash et al. 2001; Strickfaden et al. 2007), we performed a second analysis searching for clustering of weak motifs only (named $\mathrm{S}_{\mathrm{LRW}}$ analysis). 267 proteins (4.3\% of the proteome) with a score above 3.5 were identified. To reduce the number of false positives, the threshold was increased to 4.8 , a score at which the enrichment for cell cycle-related proteins was $24 \%$; similar to that obtained in the identification of Cdk1 targets in S. cerevisiae (Holt et al. 2009). This reduced the set to 175 positive proteins $(2.68 \%)$, which included $42 \%$ of the proteins identified in the $\mathrm{S}_{\mathrm{LRF}}$ analysis (Fig. 1). In sum, the combination of $\mathrm{S}_{\mathrm{LRF}}$ and $\mathrm{S}_{\mathrm{LRW}}$ allowed the identification of 228 proteins, representing the $3.6 \%$ of the $C$. albicans proteome. GO analysis revealed that in addition to cell cycle-related processes there was an enrichment in proteins belonging to other cellular processes, such as filamentous growth (33/228, 14.5\%) or transcription regulation (27/228 11.8\%). A selection of putative Cdk1 substrates grouped by GO cellular component is shown in Fig. 1. Notably, we found that proteins involved in environmental sensing were also present in the predicted Cdk substrates. Environmental sensing is a complex process that includes several steps, and putative regulatory targets were found in proteins involved in cell wall regulation (CWR), transcriptional control and cell signaling. In the following sections, we shall describe some examples to illustrate how Cdk-control mechanisms could have been integrated with other signaling pathways in Candida and that are different from those seen in S. cerevisiae. 


\subsubsection{Transcriptional regulation}

Transcription factors constitute one of the gene families enriched in pathogenic Candida species (Butler et al. 2009). In our analysis, we found several transcriptional regulators involved in environmental responses (Fig. 2A), suggesting that Cdk signaling could regulate their activity. These factors are: Sfu1 and Hap43 (iron response); Dal81 and Hsf1 (heat response); Mrr1 and Ndt80 (drug resistance); Ace2 (biofilm); Sfl1 (caspofungin response) and Mig1 and Nrg1 (yeast-tohypha transition). Comparison with their S. cerevisiae orthologs showed that five of them -Hap43, Ndt80, Hsf1, Ace2 and Nrg1- have at least one cluster with five or more Cdk sites in regions that are not present in $S$. cerevisiae. This suggests that their regulation could have additional layers of complexity in Candida. As an example, an alignment of the N-terminal region of C. albicans Hap43 with S. cerevisiae Hap4 is shown in Fig. 2B. This region contains the $\mathrm{CBC}$ domain that is required for the interaction with the Hap2/3/5 complex (Bourgarel et al. 1999), which is conserved in both proteins. Interestingly, in C. albicans the CBC domain is located in a disordered region predicted by PONDR (1 to 128), which also contains 6 weak Cdk sites not present in S. cerevisiae, suggesting that Cdk1 might modulate the interaction of Hap43 with the complex. Iron homeostasis is essential for microorganisms, such as C. albicans, that compete for iron in a mammalian host. Hap43 is essential for iron-responsive transcriptional regulation and virulence in C. albicans (Hsu et al. 2011). Iron regulatory networks have undergone a differential evolution since $S$. cerevisiae and $C$. albicans diverged, regulation in each yeast species being adapted to their specific growth conditions.

\subsubsection{Cell wall regulation}

In addition to its protective role, the cell wall plays an important function in interaction with the environment, both in sensing external cues and in interacting with other cells. There is a significant expansion of cell wall gene families in pathogenic species, suggesting that cell wall regulation could be important for virulence (Butler et al. 2009). Recently, phenotypic analysis regarding sensitivity to cell wall stresses of a collection of protein kinase mutants has shown that cell wall signaling networks in C. albicans are expanded in comparison to those of S. cerevisiae, and that some signaling pathways have been rewired and integrated in the cell wall integrity response in Candida (Blankenship et al. 2010). Five kinases related to the CWR were identified in our analysis (Fig. 2A): three proteins (Bck1, Gin4, and Mob2, this latter being the regulatory subunit of the Cbk1-Mob2 complex) with conserved roles in CWR in C. albicans and S. cerevisiae, and two (Swe1 and Hst7) with apparent C. albicans-CWR specific functions (Blankenship et al. 2010).

These kinases have high $\mathrm{S}_{\mathrm{LR}}$ scores, suggesting that Cdks might modulate their kinase activity under specific circumstances. All of them contain clusters of Cdk 
sites of different lengths located at disordered regions that are either absent in their $S$. cerevisiae orthologs or that are at different regions of the protein. The most striking example is Gin4 ( $\mathrm{S}_{\mathrm{LRF}}$ 18.34), which has an amino acid sequence with 6 contiguous strong Cdk sites $\left(\mathrm{S}^{433} ; \mathrm{S}^{447}, \mathrm{~S}^{451}, \mathrm{~S}^{455}, \mathrm{~S}^{459}\right.$ and $\left.\mathrm{S}^{463}\right)$ in a disordered region. The alignment of Gin4 with its orthologs shows some interesting aspects. First, this cluster is absent in the Saccharomyces clade (Fig. 3), although other flanking Cdk sites are more or less conserved in position. Second, in the Candida clade, an increase in the number of Cdk sites is observed from $C$. lusitaniae to $C$. albicans. Thus, this progressive accumulation of strong Cdk sites at the same disordered region suggests a linage-specific regulation of Gin4 by Cdks. The Gin4 kinase is involved in septin organization in both yeasts (Longtine et al. 1998; Wightman et al. 2004). In S. cerevisiae, Gin4 is activated during mitosis in a Cdc28/Clb2-dependent manner (Altman and Kellogg 1997). This regulation is also probably conserved in C. albicans, since Gin4 phosphorylates the septin Cdc11 at Ser395 at the end of the cell cycle (Sinha et al. 2007). It is likely that in both yeasts cell cycle regulation could be exerted through the conserved Cdk sites, while the cluster of Cdk sites in CaGin4 might create new interactions through other phosphopeptide-binding proteins. Indeed, it has been shown that a gin $4 \Delta$ mutant is hypersensitive to oxidative or osmotic stresses in C. albicans but not in S. cerevisiae (Blankenship et al. 2010).

\subsubsection{Cell signaling}

MAP kinase-mediated pathways are important stress-signaling modules essential for the development of appropriate acute and adaptative responses to environmental cues. In pathogenic fungi, these pathways are important for virulence (Román et al. 2007). Our analysis suggests that the Cek1-mediated pathway could receive inputs from Cdk signaling at different levels, since several components of the network were identified (Fig. 4). In C. albicans, this pathway is involved in mating and hyphal growth (Chen et al. 2002; Cote et al. 2011; Kohler and Fink 1996; Leberer et al. 1996; Liu et al. 1994; Yi et al. 2011). This network is composed of Cst20, the Ste11-Hst7-Cek1 module, the scaffolding protein Cst5, and the transcription factor Cph1. Finally, the Cpp1 phosphatase inhibits the pathway, probably by dephosphorylating Cek1 (Csank et al. 1997). During mating, this MAPK pathway activates different transcription factors depending on the cell type (Soll 2011)(Fig. 4). Recently, it has been shown that the exposure of C. albicans cells to $\beta$-glucan is controlled by the Cek1-mediated pathway (Galán-Díez et al. 2010), suggesting that this pathway might modulate innate immunoresponses triggered through dectin-1.

Our computational analysis also suggests that Cdks could regulate the RAM signaling pathway (Nelson et al. 2003) through the phosphorylation of Mob2 (Fig. 2 and 3). In S. cerevisiae, this pathway controls cell separation and polarized 
growth through the activity of the NDR kinase Cbk1 (Weiss et al. 2002), which requires interaction with Mob2 for its function. Whereas CaMob2 showed a $\mathrm{S}_{\mathrm{LRF}}$ value of 6.75 (the top 38), its $S$. cerevisiae ortholog had a $S_{\mathrm{LRF}}$ value of 0.77 (the top 887 from the $S$. cerevisiae proteome). This divergence suggests a new role for Cdks in the regulation of the Cbk1/Mob2 complex in C. albicans, which has been experimentally demonstrated (Gutiérrez-Escribano et al. 2011). In agreement with this idea, the cluster of four full Cdk sites $\left(S^{44} ; S^{51}, S^{67}\right.$ and $\left.S^{97}\right)$ present in the amino terminal region of CaMob2 is absent in the Saccharomyces clade (Fig. 3).

Hyphal growth is characterized by robust polarized growth at cell tips and by the inhibition of cell separation after cytokinesis. Therefore, polarized growth and cell separation, the two major outputs of the RAM pathway, are differentially regulated in yeast and hyphae. We found that hyphal-inducing cues modulate the function of Cbk1/Mob2 through Cdk-dependent phosphorylation of Mob2 in a growth-dependent manner (Gutiérrez-Escribano et al. 2011). Phenotypic analysis of cell expressing a phosphodeficient Cdk Mob2 mutant suggests a role for these types of phosphorylation in promoting maintained polarized growth and inhibiting cell separation specifically in the hyphal form but not in yeast cells.

\section{Conclusions}

Several studies have shown the existence of rewiring in C. albicans transcriptional regulatory pathways (Li and Johnson 2010) and protein kinases (Blankenship et al. 2010). Recently, we have shown that Cdk is essential to for the differential modification of the outputs of a core signaling system, the Cbk1/Mob2 complex, depending on the environmental signals that activate different cell fate programs (yeast or hypha) (Gutiérrez-Escribano et al. 2011). In this chapter we have speculated that Cdks might be connected to broader range of signaling pathways than in S. cerevisiae through the acquisition of Candida linage-specific Cdk sites clustered in proteins involved in environmental responses. Our Cdk clusterbased search in combination with other evidences, such as localization in disordered regions and the evolution of such clusters in other Hemiascomycetes, could be used to predict new layers of Cdk regulation in C. albicans environmental sensing networks.

\section{Acknowledgments}

This work was supported by grants from the Spanish Ministry of Science and Innovation to JCB (BFU2009-11251) and to CRV (BFU2010-15884) and the Regional Government of Extremadura (PRI08A017 and GRU09001) to JCB. The IBFG is institutionally supported by Fundación Ramón Areces. 


\section{References}

Altman R, Kellogg D (1997) Control of mitotic events by Nap1 and the Gin4 kinase. J Cell Biol 138:119-130

Archambault V, Buchler NE, Wilmes GM, Jacobson MD, Cross FR (2005) Two-faced cyclins with eyes on the targets. Cell Cycle 4:125-130. doi:10.1016/j.molcel.2004.05.025

Bachewich C, Whiteway M (2005) Cyclin Cln3p links G1 progression to hyphal and pseudohyphal development in C. albicans. Eukaryot Cell 4:95-102. doi:10.1128/EC.4.1.95-102.2005

Beltrao P, Serrano L (2007) Specificity and evolvability in eukaryotic protein interaction networks. PLoS Comput Biol 3:e25. doi:10.1371/journal.pcbi.0030025

Beltrao P, Trinidad JC, Fiedler D, Roguev A, Lim WA, Shokat KM, Burlingame AL, Krogan NJ (2009) Evolution of phosphoregulation: comparison of phosphorylation patterns across yeast species. PLoS Biol 7:e1000134. doi:10.1371/journal.pbio.1000134

Bensen ES, Clemente-Blanco A, Finley KR, Correa-Bordes J, Berman J (2005) The mitotic cyclins Clb2p and Clb4p affect morphogenesis in Candida albicans. Mol Biol Cell 16:33873400. doi:10.1091/mbc.E04-12-1081

Bhattacharyya RP, Remenyi A, Yeh BJ, Lim WA (2006) Domains, motifs, and scaffolds: the role of modular interactions in the evolution and wiring of cell signaling circuits. Annu Rev Biochem 75:655-680. doi:10.1146/annurev.biochem.75.103004.142710

Biswas S, Van Dijck P, Datta A (2007) Environmental sensing and signal transduction pathways regulating morphopathogenic determinants of Candida albicans. Microbiol Mol Biol Rev 71:348-376. doi:10.1128/MMBR.00009-06

Blankenship JR, Fanning S, Hamaker JJ, Mitchell AP (2010) An extensive circuitry for cell wall regulation in Candida albicans. PLoS Pathog 6:e1000752. doi:10.1371/journal.ppat.1000752

Bloom J, Cross FR (2007) Multiple levels of cyclin specificity in cell-cycle control. Nat Rev Mol Cell Biol 8:149-160. doi:10.1038/nrm2105

Bourgarel D, Nguyen CC, Bolotin-Fukuhara M (1999) HAP4, the glucose-repressed regulated subunit of the HAP transcriptional complex involved in the fermentation-respiration shift, has a functional homologue in the respiratory yeast Kluyveromyces lactis. Mol Microbiol 31:1205-1215

Braun BR, Head WS, Wang MX, Johnson AD (2000) Identification and characterization of TUP1-regulated genes in Candida albicans. Genetics 156:31-44

Braun BR, Johnson AD (1997) Control of filament formation in Candida albicans by the transcriptional repressor TUP1. Science 277:105-109

Budovskaya YV, Stephan JS, Deminoff SJ, Herman PK (2005) An evolutionary proteomics approach identifies substrates of the cAMP-dependent protein kinase. Proc Natl Acad Sci U S A 102:13933-13938. doi:10.1073/pnas.0501046102

Butler G, Rasmussen MD, Lin MF et al. (2009) Evolution of pathogenicity and sexual reproduction in eight Candida genomes. Nature 459:657-662. doi:10.1038/nature08064

Calderone RA, Fonzi WA (2001) Virulence factors of Candida albicans. Trends Microbiol 9:327-335. doi:S0966-842X(01)02094-7

Cote P, Sulea T, Dignard D, Wu C, Whiteway M (2011) Evolutionary reshaping of fungal mating pathway scaffold proteins. MBio 2. doi:10.1128/mBio.00230-10

Coudreuse D, Nurse P (2010) Driving the cell cycle with a minimal CDK control network. Nature 468:1074-1079. doi:10.1038/nature09543

Csank C, Makris C, Meloche S, Schroppel K, Rollinghoff M, Dignard D, Thomas D, Whiteway M (1997) Derepressed hyphal growth and reduced virulence in a VH1 family-related protein phosphatase mutant of the human pathogen Candida albicans. Mol Biol Cell 8:2539-2551

Chapa y Lazo B, Bates S, Sudbery P (2005) The G1 cyclin Cln3 regulates morphogenesis in Candida albicans. Eukaryot Cell 4:90-94. doi:10.1128/EC.4.1.90-94.2005 
Chen J, Lane S, Liu H (2002) A conserved mitogen-activated protein kinase pathway is required for mating in Candida albicans. Mol Microbiol 46:1335-1344. doi:10.1046/j.13652958.2002.03249.x

Echalier A, Endicott JA, Noble ME (2010) Recent developments in cyclin-dependent kinase biochemical and structural studies. Biochim Biophys Acta 1804:511-519. doi:10.1016/j.bbapap.2009.10.002

Eggimann P, Garbino J, Pittet D (2003) Epidemiology of Candida species infections in critically ill non-immunosuppressed patients. Lancet Infect Dis 3:685-702. doi:10.1016/S14733099(03)00801-6

Fisher DL, Nurse P (1996) A single fission yeast mitotic cyclin B p34cdc2 kinase promotes both S-phase and mitosis in the absence of G1 cyclins. EMBO J 15:850-860

Galán-Díez M, Arana DM, Serrano-Gómez D, Kremer L, Casasnovas JM, Ortega M, CuestaDomínguez A, Corbi AL, Plá J, Fernández-Ruiz E (2010) Candida albicans $\beta$-glucan exposure is controlled by the fungal CEK1-mediated mitogen-activated protein kinase pathway that modulates immune responses triggered through dectin-1. Infect Immun 78:1426-1436. doi:10.1128/IAI.00989-09

González-Novo A, Correa-Bordes J, Labrador L, Sánchez M, Vázquez de Aldana CR, Jiménez J (2008) Sep7 is essential to modify septin ring dynamics and inhibit cell separation during $C$. albicans hyphal growth. Mol Biol Cell 19:1509-1518. doi:10.1091/mbc.E07-09-0876

Gutiérrez-Escribano P, González-Novo A, Suárez MB, Li C-R, Wang Y, Vázquez de Aldana CR, Correa-Bordes J (2011) Cdk-dependent phosphorylation of Mob2 is essential for hyphal development in Candida albicans. Mol Biol Cell In press

Holt LJ, Tuch BB, Villen J, Johnson AD, Gygi SP, Morgan DO (2009) Global analysis of Cdk1 substrate phosphorylation sites provides insights into evolution. Science 325:1682-1686. doi:10.1126/science.1172867

Homann OR, Dea J, Noble SM, Johnson AD (2009) A phenotypic profile of the Candida albicans regulatory network. PLoS Genet 5:e1000783. doi:10.1371/journal.pgen.1000783

Hsu PC, Yang CY, Lan CY (2011) Candida albicans Hap43 is a repressor induced under lowiron conditions and is essential for iron-responsive transcriptional regulation and virulence. Eukaryot Cell 10:207-225. doi:10.1128/EC.00158-10

Huang D, Friesen H, Andrews B (2007) Pho85, a multifunctional cyclin-dependent protein kinase in budding yeast. Mol Microbiol 66:303-314. doi:10.1111/j.1365-2958.2007.05914.X

Iakoucheva LM, Radivojac P, Brown CJ, O'Connor TR, Sikes JG, Obradovic Z, Dunker AK (2004) The importance of intrinsic disorder for protein phosphorylation. Nucleic Acids Res 32:1037-1049. doi:10.1093/nar/gkh253

Kadosh D, Johnson AD (2005) Induction of the Candida albicans filamentous growth program by relief of transcriptional repression: a genome-wide analysis. Mol Biol Cell 16:2903-2912. doi:10.1091/mbc.E05-01-0073

Kohler JR, Fink GR (1996) Candida albicans strains heterozygous and homozygous for mutations in mitogen-activated protein kinase signaling components have defects in hyphal development. Proc Natl Acad Sci U S A 93:13223-13228

Leberer E, Harcus D, Broadbent ID, Clark KL, Dignard D, Ziegelbauer K, Schmidt A, Gow NA, Brown AJ, Thomas DY (1996) Signal transduction through homologs of the Ste20p and Ste7p protein kinases can trigger hyphal formation in the pathogenic fungus Candida albicans. Proc Natl Acad Sci U S A 93:13217-13222

Li H, Johnson AD (2010) Evolution of transcription networks--lessons from yeasts. Curr Biol 20:R746-753. doi:10.1016/j.cub.2010.06.056

Liu H, Kohler J, Fink GR (1994) Suppression of hyphal formation in Candida albicans by mutation of a STE12 homolog. Science 266:1723-1726

Lo HJ, Kohler JR, DiDomenico B, Loebenberg D, Cacciapuoti A, Fink GR (1997) Nonfilamentous C. albicans mutants are avirulent. Cell 90:939-949. doi:S0092-8674(00)80358-X

Loeb JD, Sepulveda-Becerra M, Hazan I, Liu H (1999) A G1 cyclin is necessary for maintenance of filamentous growth in Candida albicans. Mol Cell Biol 19:4019-4027 
Lohse MB, Johnson AD (2009) White-opaque switching in Candida albicans. Curr Opin Microbiol 12:650-654. doi:10.1016/j.mib.2009.09.010

Longtine MS, Fares H, Pringle JR (1998) Role of the yeast Gin4p protein kinase in septin assembly and the relationship between septin assembly and septin function. J Cell Biol 143:719-736

Loog M, Morgan DO (2005) Cyclin specificity in the phosphorylation of cyclin-dependent kinase substrates. Nature 434:104-108. doi:10.1038/nature03329

Massey SE, Moura G, Beltrao P, Almeida R, Garey JR, Tuite MF, Santos MA (2003) Comparative evolutionary genomics unveils the molecular mechanism of reassignment of the CTG codon in Candida spp. Genome Res 13:544-557. doi:10.1101/gr.811003

Morrison DK (2009) The 14-3-3 proteins: integrators of diverse signaling cues that impact cell fate and cancer development. Trends Cell Biol 19:16-23. doi:10.1016/j.tcb.2008.10.003

Moseley JB, Nurse P (2009) Cdk1 and cell morphology: connections and directions. Curr Opin Cell Biol 21:82-88. doi:10.1016/j.ceb.2008.12.005

Moses AM, Heriche JK, Durbin R (2007a) Clustering of phosphorylation site recognition motifs can be exploited to predict the targets of cyclin-dependent kinase. Genome Biol 8:R23. doi:10.1186/gb-2007-8-2-r23

Moses AM, Liku ME, Li JJ, Durbin R (2007b) Regulatory evolution in proteins by turnover and lineage-specific changes of cyclin-dependent kinase consensus sites. Proc Natl Acad Sci U S A 104:17713-17718. doi:10.1073/pnas.0700997104

Murad AM, d'Enfert C, Gaillardin C, Tournu H, Tekaia F, Talibi D, Marechal D, Marchais V, Cottin J, Brown AJ (2001a) Transcript profiling in Candida albicans reveals new cellular functions for the transcriptional repressors CaTup1, CaMig1 and CaNrg1. Mol Microbiol 42:981-993

Murad AM, Leng P, Straffon M, Wishart J, Macaskill S, MacCallum D, Schnell N, Talibi D, Marechal D, Tekaia F, d'Enfert C, Gaillardin C, Odds FC, Brown AJ (2001b) NRG1 represses yeast-hypha morphogenesis and hypha-specific gene expression in Candida albicans. EMBO J 20:4742-4752. doi:10.1093/emboj/20.17.4742

Nash P, Tang X, Orlicky S, Chen Q, Gertler FB, Mendenhall MD, Sicheri F, Pawson T, Tyers M (2001) Multisite phosphorylation of a CDK inhibitor sets a threshold for the onset of DNA replication. Nature 414:514-521. doi:10.1038/35107009

Nelson B, Kurischko C, Horecka J, Mody M, Nair P, Pratt L, Zougman A, McBroom LD, Hughes TR, Boone C, Luca FC (2003) RAM: a conserved signaling network that regulates Ace2p transcriptional activity and polarized morphogenesis. Mol Biol Cell 14:3782-3803. doi:10.1091/mbc.E03-01-0018

Román E, Arana DM, Nombela C, Alonso-Monge R, Plá J (2007) MAP kinase pathways as regulators of fungal virulence. Trends Microbiol 15:181-190. doi:10.1016/j.tim.2007.02.001

Santos MA, Tuite MF (1995) The CUG codon is decoded in vivo as serine and not leucine in Candida albicans. Nucleic Acids Res 23:1481-1486

Seet BT, Dikic I, Zhou MM, Pawson T (2006) Reading protein modifications with interaction domains. Nat Rev Mol Cell Biol 7:473-483. doi:10.1038/nrm1960

Serber Z, Ferrell JE, Jr. (2007) Tuning bulk electrostatics to regulate protein function. Cell 128:441-444. doi:10.1016/j.cell.2007.01.018

Sinha I, Wang YM, Philp R, Li CR, Yap WH, Wang Y (2007) Cyclin-dependent kinases control septin phosphorylation in Candida albicans hyphal development. Dev Cell 13:421-432. doi:10.1016/j.devcel.2007.06.011

Slutsky B, Staebell M, Anderson J, Risen L, Pfaller M, Soll D (1987) "White-opaque transition": a second high-frequency switching system in Candida albicans. J Bacteriol 169:189-197

Soll DR (2009) Why does Candida albicans switch? FEMS Yeast Res 9:973-989. doi:10.1111/j.1567-1364.2009.00562.x

Soll DR (2011) Evolution of a new signal transduction pathway in Candida albicans. Trends Microbiol 19:8-13. doi:10.1016/j.tim.2010.10.001 
Songyang Z, Blechner S, Hoagland N, Hoekstra MF, Piwnica-Worms H, Cantley LC (1994) Use of an oriented peptide library to determine the optimal substrates of protein kinases. Curr Biol 4:973-982. doi:S0960-9822(00)00221-9

Stoldt VR, Sonneborn A, Leuker CE, Ernst JF (1997) Efg1p, an essential regulator of morphogenesis of the human pathogen Candida albicans, is a member of a conserved class of bHLH proteins regulating morphogenetic processes in fungi. EMBO J 16:1982-1991. doi:10.1093/emboj/16.8.1982

Strickfaden SC, Winters MJ, Ben-Ari G, Lamson RE, Tyers M, Pryciak PM (2007) A mechanism for cell-cycle regulation of MAP kinase signaling in a yeast differentiation pathway. Cell 128:519-531. doi:10.1016/j.cell.2006.12.032

Sudbery P, Gow N, Berman J (2004) The distinct morphogenic states of Candida albicans. Trends Microbiol 12:317-324

Ubersax JA, Woodbury EL, Quang PN, Paraz M, Blethrow JD, Shah K, Shokat KM, Morgan DO (2003) Targets of the cyclin-dependent kinase Cdk1. Nature 425:859-864. doi:10.1038/nature02062

Wang A, Lane S, Tian Z, Sharon A, Hazan I, Liu H (2007) Temporal and spatial control of HGC1 expression results in Hgc1 localization to the apical cells of hyphae in Candida albicans. Eukaryot Cell 6:253-261. doi:10.1128/EC.00380-06

Wang A, Raniga PP, Lane S, Lu Y, Liu H (2009) Hyphal chain formation in Candida albicans: Cdc28-Hgc1 phosphorylation of Efg1 represses cell separation genes. Mol Cell Biol 29:44064416. doi:10.1128/MCB.01502-08

Wang Y (2009) CDKs and the yeast-hyphal decision. Curr Opin Microbiol 12:644-649. doi:10.1016/j.mib.2009.09.002

Weiss EL, Kurischko C, Zhang C, Shokat K, Drubin DG, Luca FC (2002) The Saccharomyces cerevisiae Mob2p-Cbk1p kinase complex promotes polarized growth and acts with the mitotic exit network to facilitate daughter cell-specific localization of Ace2p transcription factor. J Cell Biol 158:885-900. doi:10.1083/jcb.200203094

Whiteway M, Bachewich C (2007) Morphogenesis in Candida albicans. Annu Rev Microbiol 61:529-553. doi:10.1146/annurev.micro.61.080706.093341

Wightman R, Bates S, Amornrrattanapan P, Sudbery P (2004) In Candida albicans, the Nim1 kinases Gin4 and Hsl1 negatively regulate pseudohypha formation and Gin4 also controls septin organization. J Cell Biol 164:581-591. doi:10.1083/jcb.200307176

Yi S, Sahni N, Daniels KJ, Lu KL, Huang G, Garnaas AM, Pujol C, Srikantha T, Soll DR (2011) Utilization of the mating scaffold protein in the evolution of a new signal transduction pathway for biofilm development. MBio 2:e00237-00210. doi:10.1128/mBio.00237-10

Zheng X, Wang Y (2004) Hgc1, a novel hypha-specific G1 cyclin-related protein regulates Candida albicans hyphal morphogenesis. EMBO J 23:1845-1856. doi:10.1038/sj.emboj.7600195

Zheng XD, Lee RT, Wang YM, Lin QS, Wang Y (2007) Phosphorylation of Rga2, a Cdc42 GAP, by CDK/Hgc1 is crucial for Candida albicans hyphal growth. EMBO J 26:3760-3769. doi:10.1038/sj.emboj.7601814 


\section{FIGURE LEGENDS}

Fig. 1. Selected proteins with Cdk1 regulatory clusters grouped by GO cellular process. The color of the box indicates whether a protein was identified in the strong phosphorylation site search $\left(\mathrm{S}_{\mathrm{LRF}}\right)$, in the weak phosphorylation site analysis $\left(\mathrm{S}_{\mathrm{LRW}}\right)$ or in both. Proteins in red show Cdkdependent phosphorylation in S. cerevisiae and/or C. albicans (Ubersax et al., 2003; Beltrao et al., 2009; Holt et al., 2009). The inset shows a graphic representation of the results obtained in the SLRF or SLRW analysis.

Fig. 2. (A) Predicted Cdk1 substrates in cell wall and transcriptional regulation. In the cell wall regulation pathway, many protein kinases involved in CWR or the regulatory subunit of the Cbk1-Mob2 complex contain putative Cdk1 regulatory clusters. Cdks can also control transcription activation or repression through the phosphorylation of transcription factors in different pathways. (B) Alignment of the N-terminal region of C. albicans Hap43 and S. cerevisiae Hap4. Weak Cdk sites are indicated in blue, while the rectangle shows the conserved CBC domain.

Fig. 3. Evolution of clusters of Cdk regulatory sites in Hemiascomycota: examples of the appearance of clusters of regulatory sites in the Candida clade. At the top, a schematic representation of Mob2 and Gin4 with their different domains. Strong (red) and weak (blue) Cdk phosphorylation sites, regulatory regions predicted by SLR (dark grey) and disordered regions (brackets below the sequence) are also indicated. The sequence of the regions containing the predicted regulatory clusters (indicated with dashed lines) is aligned below with their orthologs from the Candida and Saccharomyces clades. The dashed line indicates gaps introduced to maximize the alignment.

Fig. 4. The Cph1-mediated MAPK pathway can be regulated by Cdk1 at different levels. Putative Cdk1 regulatory clusters are present in different components of the Cst20-Cek1 pathway and in two transcriptional repressors. To the right, a schematic representation of the six putative targets of the pathway, indicating the different domains in each protein, the position of the Cdk phosphorylation sites, the putative regulatory regions, and disordered regions. 
Chromatin structure DNA replication

orf19.1253 (ScPho4) orf19.4301 (ScSpt21) orf19.470 (ScTaf12) orf19.5105 (ScGal11) orf19.5799 (ScCaf120) Rpo21

Tel1

\section{Orc1}

Orc2

orf19.4412 (ScRev1) orf19.6155 (ScCdc9) orf19.6291 (ScFun30) orf19.652 (ScYen1) orf19.7023 (Scloc4) Rad9

\begin{tabular}{|ll|}
\hline Orc6 $\quad$ Pif1 \\
Tfg1
\end{tabular}

Cell Polarity orf19.177 (ScBem1) Bni4

Boi2

\begin{tabular}{|l|l|}
\hline Ase1 & Bud14 \\
Bni1 & Chs4 \\
Cyk3 & Gin4 \\
Hof1 & Iqg1 \\
Int1 & Rga2 \\
Mob2 & Spa2 \\
Rgd2 & \\
Rgd3 & \\
\hline
\end{tabular}

Transcriptional activators

\begin{tabular}{|lll|l|}
\hline orf19.3407 (Sc Rad18) & Ace2 \\
Brg1 & Hap43 & Sfl1 & Ash1 \\
Cta8 & Mrr1 & Sfu1 & Fkh2 \\
Dal81 & Ndt80 & Sko1 & Hcm1 \\
Fhl1 & Sef1 & Swi1 & \\
Gzf3 & & & Zcf20 \\
& & &
\end{tabular}

Transcriptional Signal Transduction Cell Cycle represors

\begin{tabular}{|l|}
\hline Mig1 \\
Nrg1 \\
Rxt3 \\
Ume6 \\
\hline Yox1 \\
\hline
\end{tabular}

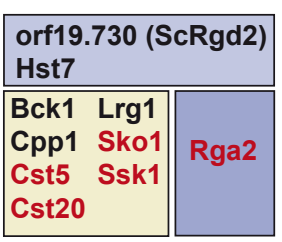

\begin{tabular}{|c|c|}
\hline \multicolumn{2}{|c|}{ orf19.267 (ScNet1) } \\
\hline $\begin{array}{l}\text { Cln3 } \\
\text { Hgc1 } \\
\text { Mih1 }\end{array}$ & $\begin{array}{l}\text { Smc2 } \\
\text { Smc3 }\end{array}$ \\
\hline $\begin{array}{l}\text { Sol1 } \\
\text { Swe1 }\end{array}$ & $\begin{array}{l}\text { Cdh1 } \\
\text { Cdc6 }\end{array}$ \\
\hline
\end{tabular}

Response to

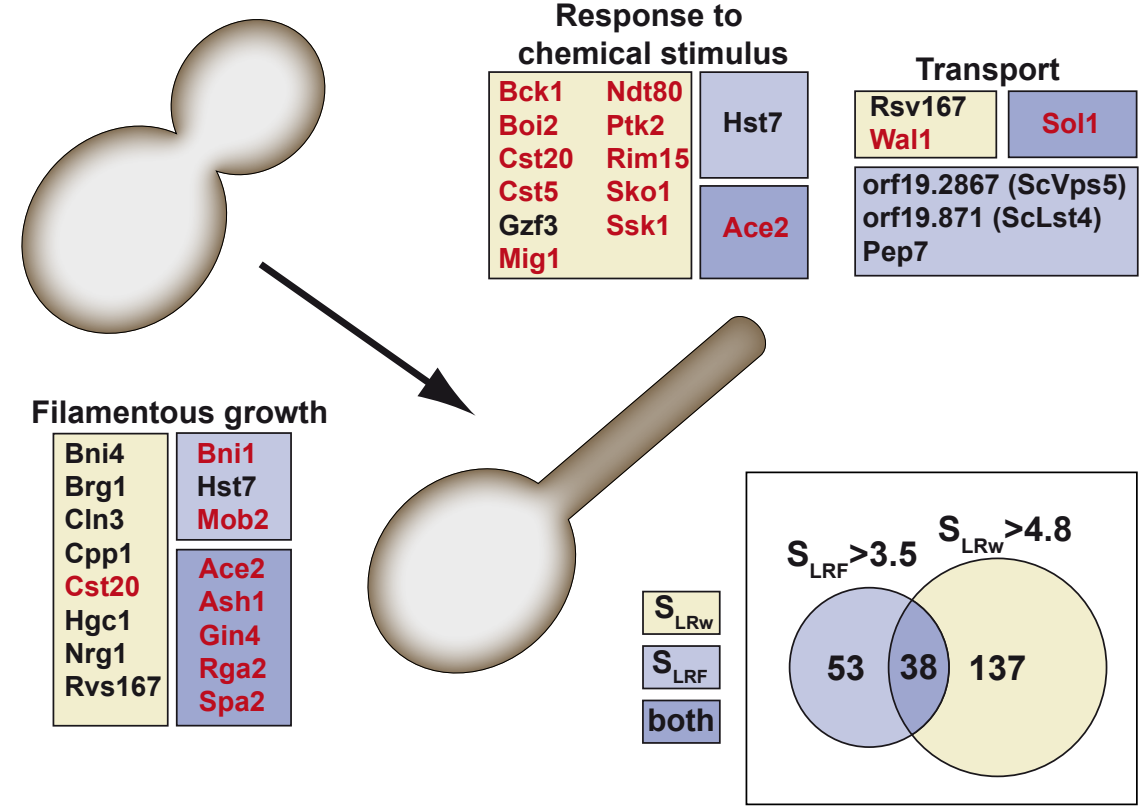


A

Cell wall biogenesis (PK cluster I)

Cbk1 Mob2

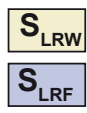

both

Repressors $\mathrm{Y} / \mathrm{H}$

\begin{tabular}{|l|l|}
\hline Mig1 & Nrg1 \\
\hline
\end{tabular}

Stress response

\begin{tabular}{|l|l|l|}
\hline Sfu1 & Sfl1 & Hsf1 \\
\hline Hap43 & Dal81 \\
\hline
\end{tabular}

Cell wall signalling

(PK cluster IV)

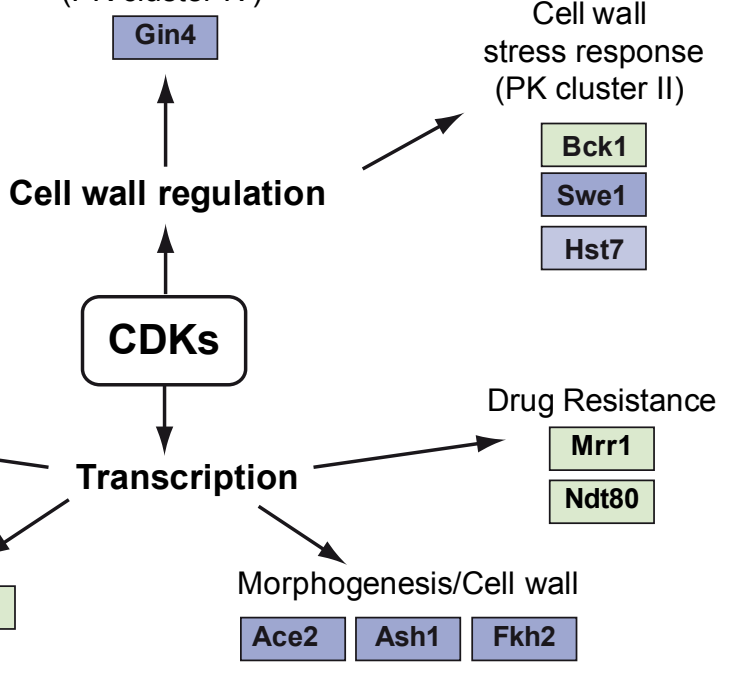

Hap43 MPAKGPNIIP-KQAPSPIAIAGSPPSSSASTPRSAASPPVSVNTKYSPNTNSNTIMPRQV 59 Hap4 MTAKTFLLQASASRPRSNHFKNEHNNI PLAPVPIAPNTNHHNNSSLEFENDGSKKKKKSS 60

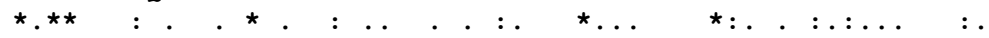

Hap43 MSIQTSKEWVLPPRPKPGRKPSVDTPASKRKAQNRAAQRAFRERRATRVQELEQKLMEVE 119 Hap4 LVVRTSKHWVLPPRPRPGRRSS SHNTLPANNTNN--ILNVGPNSRNSSNNNNNNNIISNR 118

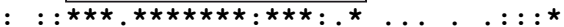




\section{Gin4}

I $S / T-P$

I $S / T-P-X-R / K$

$\sqcup$ Disordered regions

- Regulatory regions

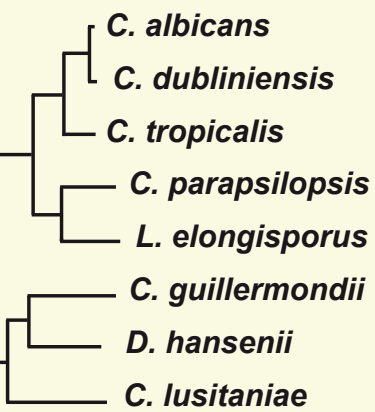

S. cerevisiae S. paradoxus

S. mikatae

S. bayanus

C. glabrata

K. lactis

A. gossypii

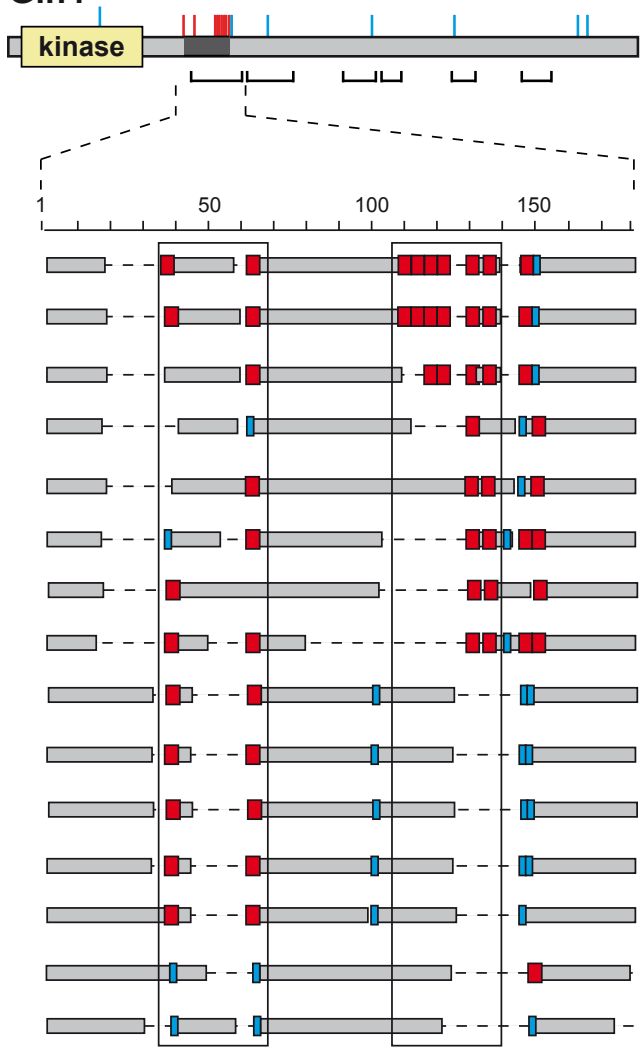

\section{Mob2}

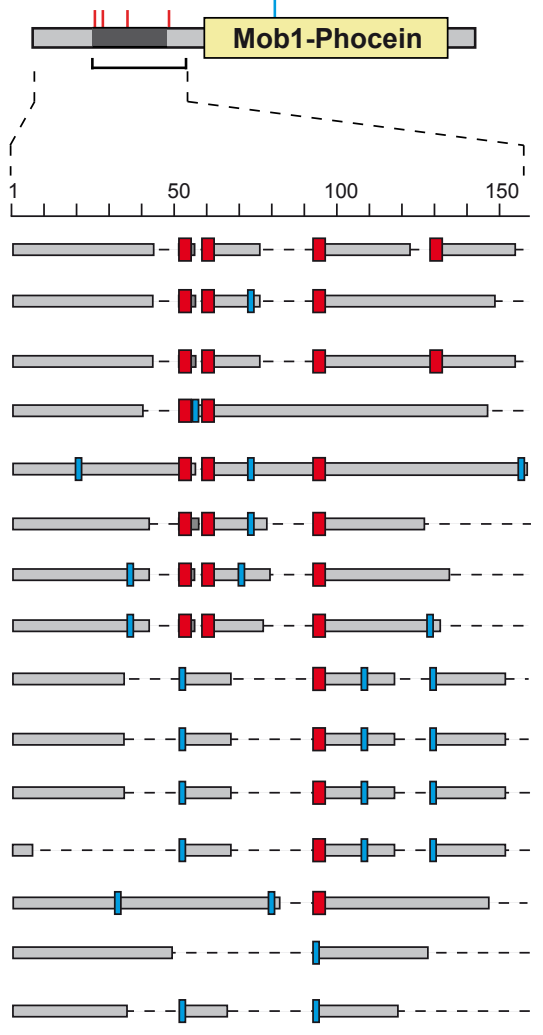




\section{INPUTs Osmotic Starvation}

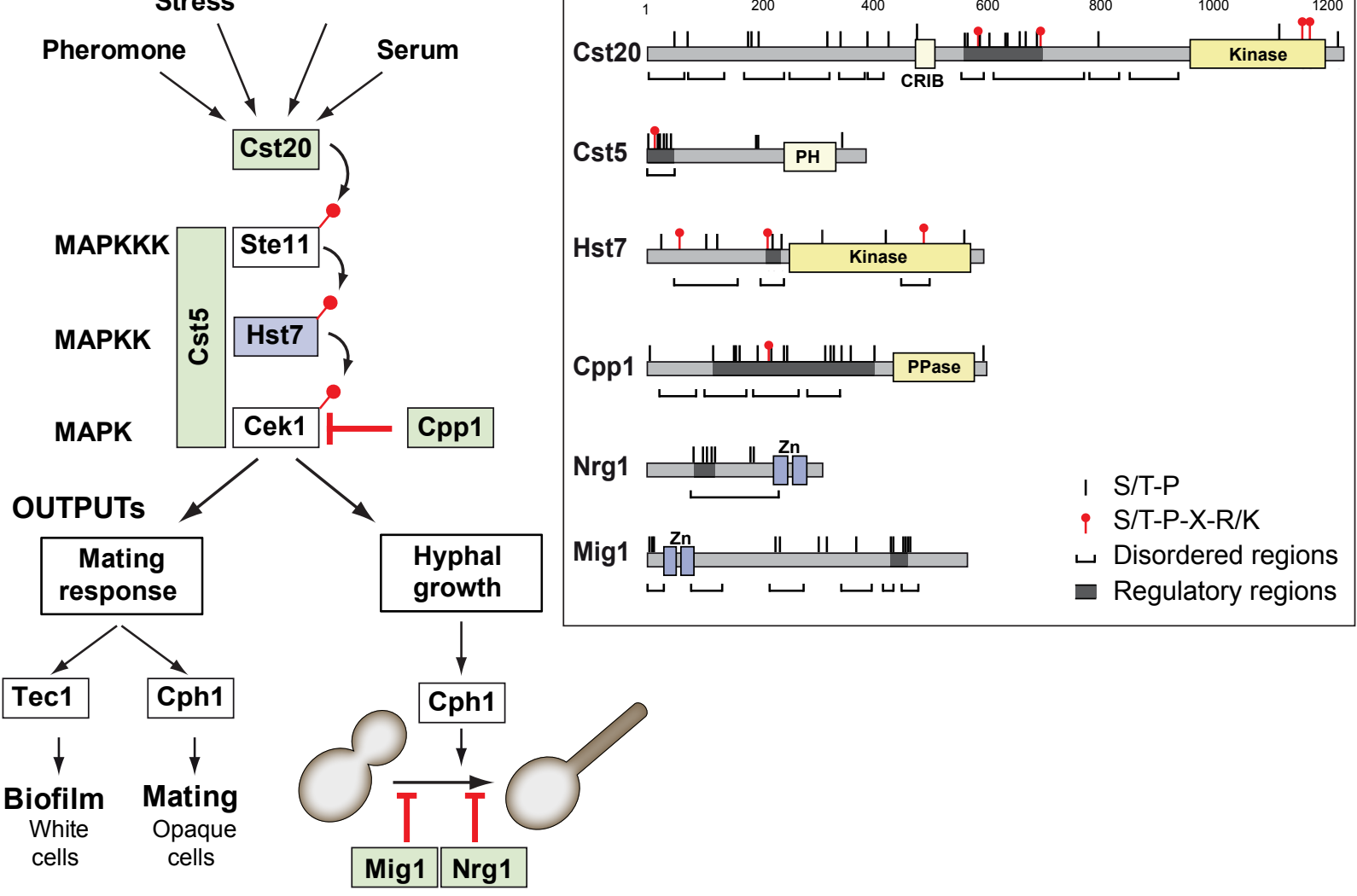

